www.jmscr.igmpublication.org

Impact Factor 5.84

Index Copernicus Value: 83.27

ISSN (e)-2347-176x ISSN (p) 2455-0450

crossref DOI: _https://dx.doi.org/10.18535/jmscr/v5i6.94

Journal Of Medical Science And Clinical Research

\title{
Ratio of Atherogenic and Antiatherogenic Lipoproteins in RVO
}

\author{
Authors \\ Dr Samreen Rafique Shaikh, Dr Pooja Nitin Joshi, Dr Arif Ansar Shaikh \\ Department of Biochemistry B J Government Medical College Pune \\ Corresponding Author \\ Dr Samreen Rafique Shaikh \\ Address:706 Guruwar Peth Master Residency Society. Pune 411042 \\ Contact number: 8793612004/9511815799, Email: drsamreenshaikh24@yahoo.com
}

\begin{abstract}
Retinal vein occlusion is the most common retinal vascular disease after diabetic retinopathy. Due to its multifactorial nature, diagnosis and management remains a challenge. Hyperlipidemia is one of the risk factors. So a case control study was undertaken to determine levels of serum lipids and ratios of atherogenic to antiatherogenic lipoproteins. Total 60 patients of the study were divided into group A (30 patients of fundoscopically diagnosed RVO) and Group B (30 age and sex matched controls). The levels of Total Cholesterol, Triglyceride, Low Density Lipoprotein, Very Low Density Lipoprotein, High Density Lipoprotein and ratios of atherogenic $(T G, L D L)$ to antiatherogenic $(H D L)$ lipoproteins were measured. The results obtained in these patients were compared to those of controls. Data analysis was performed using Graph Pad Instat software, Version 3.05. A highly significant increase in serum levels of TG, VLDL-C ( $p<0.001)$, and a significant increase in the serum levels $T C$ and $L D L-C(p<0.05)$ was found in group $A$ as compared to that of group B. However no significant decrease was found in the serum levels of HDL-C $(p>0.05)$ in group $A$ as compared to that of group $B$. There was highly significant increase in TG/HDL ratio $(p<0.001)$ and significant increase in LDL/HDL ratio $(p<0.05)$. The study concluded that elevated ratios of TG/HDL and LDL/HDL may contribute to the pathogenesis and progression of RVO and lowering the levels of these parameters may prevent the further complications of $R V O$.
\end{abstract}

Keywords: RVO, atherosclerosis, Triglycerides, Low density lipoprotein, High density lipoprotein, small density lipoproteins.

\section{Introduction}

Retinal vein occlusion (RVO) is the second commonest cause of reduced vision due to retinal vascular disease after diabetic retinopathy ${ }^{1}$. RVO presents mainly in older individuals, over $50 \%$ of cases occurring in persons older than 65 years ${ }^{2}$.

Conditions that may predispose to retinal vein occlusion include diabetes mellitus, hypertension, hyperlipidemia, systemic vascular diseases, open angle glaucoma, hyperviscosity, increased erythrocyte sedimentation, certain medications, smoking, drinking etc.

Retinal vein occlusion has been classified into 3 varieties:

1) Branch retinal vein occlusion, 2) Central retinal vein occlusion \& 3)Hemi-retinal vein occlusion ${ }^{3,4}$. 
Patients with RVO present with painless, decreased vision, complete loss of vision, or a blind spot in their visual field.

Although multiple factors, both systemic and ocular, are apparently involved in the production of this retinal vascular accident, the exact etiology of the retinal vein occlusion remains unclear. The condition may be due to either one or a combination of systemic changes known as Virchow's triad:

a) Hemodynamic changes (venous stasis),

b) Degenerative changes of the vessel wall and

c) Blood hypercoagulability. ${ }^{5}$

Hyperlipidemia is one of the factor causing degenerative changes leading to the atherosclerotic changes in the retinal artery. As CRVO, typically occurs at the lamina cribrosa where the vein and the artery share a common adventitial sheath and BRVO develops at the arteriovenous crossing sites where artery passes anterior to the vein, the arteriosclerosis related rigid and thick arterial wall causes mechanical compression of the vein resulting in turbulent blood flow in the vein thereby producing injury to the venous endothelium that leads to occlusion of the vein. ${ }^{6,7,8}$

Association of hyperlipidemia mainly hypercholesterolemia and hypertriglyceridemia and RVO is well established. ${ }^{9}$ However lipoprotein related risk depends upon overall balance between atherogenic and antiatherogenic lipoproteins. To know the effective risk, present study was undertaken to determine levels of (LDL-C, VLDL-C, HDL-C, TG, TC) and ratios of TG/HDL and LDL/HDL in patients of RVO. As lipoproteins TG and LDL are atherogenic and HDL is antiatherogenic.

\section{Material and Methods}

This study was conducted as a case control study. The study protocol was approved by the Ethical Committee of the Institute. Informed written consent was obtained from all the study subjects enrolled in the study.
The total number of study subjects included was 60. All the subjects included were above 20 years of age. Detailed clinical history and relevant clinical examination data and written consent were obtained from all subjects after explaining the study procedure. The 60 subjects were divided into two groups.

Group A: Consists of 30 FFA diagnosed patients of Retinal Vein Occlusion.

Group B: 30 age and sex matched healthy controls without Retinal vein Occlusion.

\section{Inclusion criteria}

- Age- cases of RVO between 20 to 80 years diagnosed on routine fundus evaluation,

- Sex- Both male and female,

- Patients willing to give consent

\section{Exclusion criteria}

- Patients on Lipid lowering drugs, anticoagulants,

- Anemic patients, Smokers,

- Women taking oral contraceptive pills,

- Patients of Ocular trauma,

- Patients with CRAO diagnosed on fundus examination,

- Patients not giving consent.

\section{Collection of Blood Samples}

Study details were explained to the subjects. Informed written consent was taken. Clinical examination was done as per the proforma and blood samples were collected. $5 \mathrm{ml}$ of 12 to 14 hours fasting venous sample was withdrawn from the anti-cubital vein of each participant after taking all aseptic precautions using sterile needles and syringes. Serum was separated after centrifuging. Serum was used for estimation of lipid profile. The blood samples were analyzed immediately and those samples that were not processed immediately were capped and stored at $-20^{0} \mathrm{C}$ until further analysis. The serum total cholesterol $^{10}$, HDL- cholesterol $^{11}$, triglycerides ${ }^{11}$ were determined and from that value of VLDL cholesterol (TG/5) was calculated. LDL cholesterol $^{12,13}$ was derived by FredricksonFriedewald Equation. 
All the calculations were done using Microsoft Office Excel 2010 and statistical analysis was done using the Graph Pad Instat software, Version 3.05. All statistical data was analysed by Student's unpaired, two tailed t-test. P-value less than 0.05 $(\mathrm{P}<0.05)$ was considered to be statistically significant $(\mathrm{S})$. P value of less than 0.001 ( $\mathrm{P}<$ $0.001)$ was considered to be statistically highly significant (HS). P-value more than 0.05 (P > $0.05)$ was considered to be statistically nonsignificant (NS).

\section{Results}

\section{Lipid profile in patients with RVO}

In the RVO group, Total Cholesterol was raised in $11(36.66 \%)$ patients, VLDL was found to be elevated in $15(50 \%)$ patients while triglycerides was elevated in $17(56.66 \%)$ patients, LDL was found to be elevated in 13(43.33\%) patients and HDL was decreased in $9(30 \%)$ patients in the study.

In this study, there was a highly significant increase in the serum levels of TG, VLDL-C $(<0.001)$, and a significant increase in the serum levels TC and LDL-C $(<0.05)$ in group A as compared to that of group B. However there was no significant decrease in the serum levels of HDL-C $(>0.05)$ in group A as compared to that of group B. To see the effective load of atherogenic factors the ratio of TG/HDL and LDL /HDL was taken. It was found that there was highly significant increase in TG/HDL ratio $(\mathrm{p}<0.001)$ and significant increase in LDL/HDL ratio $(\mathrm{p}<0.05)$.

Table 1: Comparison of lipid profile parameters in all groups

\begin{tabular}{|l|c|c|c|}
\hline Parameter & $\begin{array}{c}\text { Group A }(\mathrm{n}=30) \\
(\text { mean } \pm \mathrm{SD})\end{array}$ & $\begin{array}{c}\text { Group B }(\mathrm{n}=30) \\
(\text { mean } \pm \mathrm{SD})\end{array}$ & P value \\
\hline Total cholesterol $(\mathrm{mg} / \mathrm{dl})$ & $197.8+31.78$ & $176.67 \pm 33.62$ & $<0.05(\mathrm{~S})$ \\
\hline LDL- cholesterol $(\mathrm{mg} / \mathrm{dl})$ & $131.07 \pm 28.25$ & $113.57 \pm 23.48$ & $<0.05(\mathrm{~S})$ \\
\hline HDL-cholesterol $(\mathrm{mg} / \mathrm{dl})$ & $40.63+12.06$ & $45.86+10.39$ & $>0.05(\mathrm{NS})$ \\
\hline Triglycerides $(\mathrm{mg} / \mathrm{dl})$ & $174.07+47.10$ & $125.0 \pm 56.69$ & $<0.001(\mathrm{HS})$ \\
\hline VLDL-cholesterol $(\mathrm{mg} / \mathrm{dl})$ & $46.53+17.39$ & $31.76+13.80$ & $<0.001(\mathrm{HS})$ \\
\hline TG/HDL & $4.79+2.15$ & $2.94+1.90$ & $<0.001(\mathrm{HS})$ \\
\hline LDL/HDL & $3.60 \pm 1.64$ & $2.70 \pm 1.25$ & $<0.05(\mathrm{~S})$ \\
\hline
\end{tabular}

$\mathrm{S}(\mathrm{P}<0.05)=$ significant, HS $(\mathrm{P}<0.001)=$ highly significant, $\mathrm{NS}(\mathrm{P}>0.05)$ $=$ not significant

\section{Discussion}

Retinal vein occlusion (RVO) is the most common retinal vascular disease after diabetic retinopathy. ${ }^{1}$ Due to its multifactorial nature, diagnosis and management of this condition remains a challenge. Most patients develop the disease at an elderly age, and more than half of them have associated systemic disorders (e.g. hypertension, hyperlipidemia and/or diabetes mellitus). ${ }^{14}$ Degenerative changes in vessel wall is one of the factor from Virchow's triad that contributes to RVO. Many factors are responsible for causing degenerative changes and increased levels of lipids is one of them. Hyperlipidemia causes atherosclerotic changes in the blood vessel thereby predisposing to retinal vein occlusion. Considering the above facts in the present study levels of serum total cholesterol, Triglyceride, LDL-C, VLDL-C, HDL-C were determined and ratios of TG/HDL and LDL/HDL were calculated, in Fundus Fluorescein Angiography (FFA) diagnosed cases of retinal vein occlusion and the results were compared with the controls. It was observed that the levels of serum total cholesterol, Triglyceride, LDL-C, VLDL-C, and HDL-C were significantly altered in retinal vein occlusion patients.

In this study total cholesterol levels were significantly elevated in group A as compared to group $\mathrm{B}(\mathrm{P}<0.05)$ as is also observed in previous study ${ }^{15,16}$. On the contrary uncertainty in relationship of hypercholesterolemia and RVO was noted in certain studies ${ }^{17,18}$.

The mean serum triglyceride levels in group $\mathrm{A}$ and in group B were compared. Group A had significantly higher triglyceride level as compared with group B $(p<0.001)$. These findings coincide well with some previous study ${ }^{9,18}$. Similarly, the mean serum VLDL cholesterol levels of Group A were significantly higher as compared to group B (control) $(\mathrm{p}<0.001)$.Our study results are similar with a previous study ${ }^{9}$. However few studies found no significant increase in serum VLDL ${ }^{15,16}$. LDL-C and HDL-C are two lipoproteins involved in transport of cholesterol from liver to peripheral 
tissues and from peripheral tissues to liver respectively ${ }^{12}$.

In the present study the mean serum LDL cholesterol levels of group A were significantly higher as compared to group B $(p<0.05)$.These findings are similar with certain studies ${ }^{9}$. However some studies found no significant relationship of LDL-C and RVO ${ }^{15,16,17 .}$

HDL particles induce the removal of cholesterol from cells as well as atherosclerotic plaques and transport them to the liver. Along with the reverse cholesterol transport, HDL particles seem to have anti-inflammatory and antioxidant properties, inhibiting the oxidation of LDL cholesterol and expression of cellular adhesion molecules and monocyte recruitment. Thus HDL may also reduce the risk of thrombosis by inhibiting platelet activation and aggregation. Thereby conferring protection from atherosclerosis. In this study the mean serum HDL cholesterol levels of group A and group B were compared. Serum HDL cholesterol levels in group A were towards the lower side of the normal range, however it was not statistically

significant $(p>0.05)$. These findings coincide well with previous study ${ }^{15,16}$. However certain previous study, found significant decrease in serum $\mathrm{HDL}^{17}$.

This means that the levels of atherogenic lipoproteins were found to be elevated than antiatherogenic. To know effective lipoprotein related risk the ratios of TG/HDL and LDL/HDL were calculated. These ratios are better markers of atherosclerotic changes which lead to narrowing of vessel and thus restricting blood supply. It was observed in the present study that the ratio of LDL/HDL was significantly raised in group A as compared to group B $(p<0.05)$ and the ratio of TG/HDL was highly significant in group A than group B ( $p<0.001)$.Thus the study results indicate that in patients of RVO the load of atherogenic lipoprotein is much more than that of antiatherogenic. It is known that there is positive correlation between elevated ratio of TG/HDL and concentration of small dense LDL particles ${ }^{19}$. These particles penetrate the wall more easily and retain there for a longer time. ${ }^{20}$ Thus predisposing the wall for further atherogenic changes. As per our results significantly increased TG/HDL ratio indicates that there is large concentration of small dense particles. Thus efforts towards lowering these ratios will prevent the progression of RVO to further complications like macular edema, followed by retinal neovascularization, vitreous hemorrhage, or retinal detachment. It will also prevent the recurrence of RVO in the same eye or in the opposite eye. Measurement of these ratios also gives a guide to the management of RVO in patients with impending RVO or at risk of RVO .Thus evaluation of a patient of RVO should include serum lipid profile, TG/HDL, LDL/HDL in addition to ocular and medical examination. The derangement in these parameters in patients with RVO may potentially contribute to an increased risk of cardiovascular disease and stroke and lowering the levels of these parameters will prevent the occurrence.

\section{Conclusions}

It can be concluded from the present study that the ratio of atherogenic to anti-atherogenic lipoproteins that is TG/HDL and LDL/HDL may relate to the initiation of $\mathrm{RVO}$ and its further progression. Thus assessment of these ratios in the patients of RVO may give us a guide to the diagnosis, monitoring progression and management of RVO.

\section{Acknowledgement}

Department of Ophthalmology, Clinical staff of Biochemistry.

\section{References}

1. Clarkson J G: Central retinal vein occlusion. In: Ryan S3, ed. Retina.Vol.2: Medical Retina St. Louis: CV Mosby, 1989; chap 74;p.421-426

2. Hayreh SS, Zimmerman B, McCarthy MJ, Podhajsky P. Systemic diseases associated 
with various types of retinal vein occlusion. Am J Ophthalmol 2001;131(1): 6177.

3. Zegarra H, Gutaman F A, Conforto J, The natural course of central retinal vein occlusion. Ophthalmology.1979; 86(11):1931-42

4. Sekimoto M, Hayasaka S, Setogawa T. Type of Arteriovenous crossing at the site of BRVO. Jpn J Ophthalmol, 1992;36(2): 192-6.

5. Yau JW, Lee P, Wong TY, Best J, Jenkins A. Retinal vein occlusion: an approach to diagnosis, systemic risk factors and management. Intern Med J 2008; 38(12): 904-10.

6. Jefferies $\mathrm{P}$, Clemett $\mathrm{R}$, Day $\mathrm{T}$. An anatomical study of retinal arteriovenous crossings and their role in the pathogenesis of retinal branch vein occlusions. Aust N Z J Ophthalmol 1993; 21(4): 213-7.

7. Gunther J, Ingrid U. Scott, and Michael Ip. Retinal Venous Occlusive Disease.In: Albert DM, Miller JW, Azar DT, Blodi BA editors. Albert: Albert \& Jacobiec's Principles and Practice of Ophthalmology. $3^{\text {rd }}$ edition. Philadelphia. Saunders Elsevier ; 2008;p.1755- 1773.

8. Zhao J, Sastry SM, Sperduto RD, Chew EY, Remaley NA. Arteriovenous crossing patterns in branch retinal vein occlusion. Ophthalmology 1993; 100(3): 423-8.

9. DodsonP M, GaltonD J,HamiltonA $\mathrm{M}$ and BlachR K.Retinal vein occlusion and the prevalence of lipoprotein abnormalities. British Journal of Ophthalmology, 1982; 66: 161-164.

10. Tarbutton PN, Gunter LR. Clin Chem. 1974; 20:724.

11. Buccolo G, David M.Clin.Chem. 1973;19:476.

12. Rifai N, Warnick GR. Lipids, lipoproteins, apolipoproteins, and other cardiovascular risk factors. In: Burtis CA, Ashwood ER, Bruns DE, editors. Tietz textbook of clinical chemistry and molecular diagnostics. 4th ed. New Delhi: Saunders
Elsevier; 2006. p. 903-81.

13. Friedewald WT, Levy RI, Fredrickson DS. Estimation of the concentration of lowdensity lipoprotein cholesterol in plasma, without use of the preparative ultracentrifuge. ClinChem 1972;18: 499-502.

14. Wong TY, Larsen EK, Klein R, Mitchell P, Couper DJ, Klein BE et al. Cardiovascular risk factors for retinal vein occlusion and arteriolar emboli: the Atherosclerosis Risk in Communities \& Cardiovascular Health studies. Ophthalmology 2005; 112(4): 540-547.

15. Marcucci R, Bertini L, Giusti B, Brunelli T, Fedi S, Cellai AP et al. Thrombophilic risk factors in patients with central retinal vein occlusion. Thromb Haemost. 2001;86(3):772- 776.

16. Weger M, Renner W, Steinbrugger I, Cichocki L, Temmel W, Stanger O, et al. Role of thrombophilic gene polymorphhisms in branch retinal vein occlusion. Ophthalmology. 2005;112(11):1910-1915.

17. Dodson P M, Kubicki A J, Taylor K G, AND Kritzinger E E. Medical conditions underlying recurrence of retinal vein occlusion. British Journal of Ophthalmology, 1985; 69(7): 493-496.

18. Weiser M, Serog P, Apfelbaum M, Rousselie F. Incidence of lipid changes in rhegmatogenous retinal detachment, retinal vein occlusion and primary chronic glaucoma. J Fr Ophthalmol.1985;8(10): 619-22.

19. Maruyama, C., Imamura, K., and Teramoto, T. Assessment of LDL Particle Size by Triglyceride/HDL Cholesterol Ratio in Non-diabetic Healthy Subjects without Prominent Hyperlipidemia. J Atheroscler Thromb. 2003; 10: 186-191

20. Ip S, Lichtenstein AH, Chung M, Lau J, Balk EM. Systematic review: association of low- density lipoprotein subfractions with cardiovascular outcomes. Ann Intern Med. 2009;150:474-84. 


\footnotetext{
Abbreviations Used

$\%$ - Percentage

$>$ - More than

\pm - plus or minus

$\geq$ - Greater than or equal to

$\uparrow$ - Increase

$\downarrow$ - Decrease

BRVO - branch retinal vein occlusion Cunesterified cholesterol

CHOD - cholesterol oxidase

CRVO- central retinal vein occlusion HDL - High density lipoprotein LDL - Low Density Lipoprotein $\mathrm{mg} / \mathrm{dl}$ - milligram per deciliter $\mu \mathrm{g} / \mathrm{dl}$ - microgram per deciliter $\mathrm{mmol} / \mathrm{L}$ milimole per litre $\mathrm{ng} / \mathrm{ml}$ - nanogram per milliliter Opd- outpatient department TG - Triglycerides TC - Total cholesterol

VLDL - Very Low Density Lipoprotein
} 